\title{
Sufficient optimality conditions for a bang-bang trajectory in a Bolza problem.
}

\author{
Laura Poggiolini, Marco Spadini \\ Dipartimento di Matematica Applicata "G. Sansone" \\ Università segli Studi di Firenze \\ In: Mathematical Control Theory and Finance. \\ Editors: A. Sarychev; A. Shiryaev; M. Guerra M. Grossinho. \\ Pagg 337-358, Springer, Berlin. 978-3-540-69531-8 .
}

This paper gives sufficient conditions for a class of bang-bang extremals with multiple switches to be locally optimal in the strong topology. The conditions are the natural generalizations of the ones considered in the case when only simple switches occur. We require both the strict bang-bang Legendre condition, a non degeneracy condition at multiple switching times, and the second order conditions for the finite dimensional problems obtained by moving the switching times of the reference trajectory.

Keywords: Minimum time; Bangbang control; Sufficient conditions; Second variation; Hamiltonian methods 